\title{
Endolumbal nootropic-ozone therapy in complex treatment of patients with complicated spinal injury in acute period
}

\author{
Yuldashev S. S. \\ Neurology department of RSCUMA, Samarkand State Medical Institute, Samarkand, Uzbekistan \\ Email address: \\ x.karabaev@yandex.ru(Yuldashev S. S.)
}

To cite this article:

Yuldashev S. S.. Endolumbal Nootropic-Ozone Therapy in Complex Treatment of Patients with Complicated Spinal Injury in Acute Period. American Journal of Psychiatry and Neuroscience. Vol. 1, No. 1, 2013, pp. 1-4. doi: 10.11648/j.ajpn.20130101.11

\begin{abstract}
Spinal trau ma occurs when there is damage to the spinal cord that results in a bruise, a partial tear, or a complete tear. Because the spinal cord is the central carrier of signals throughout the body, damage to the spinal cord can have serious consequences. During a 5 - year period (2005 - 2009), 39 patient's spinal trauma - were treated in the Neurology department of RCSUMA. The patients were divided into two groups: The first group (main) included 25 patients, spinal injury complicated by different localization. The main criterion for evaluating the effectiveness of this method is clinic neurologic, liquor dynamic and liquorlogical: the emergence of positive neurological symptoms within two weeks after endolumbal nootropic-ozone therapy. 16 patients (64.0\%) have after 2-3 times endolumbal nootropic-ozone therapy has appeared positive neurological symptoms as return of sensation the below level of damage and slight movement in the limbs. In $6(24.0 \%)$ patients improved neurological symptoms occurred after 5-6 times the endolumbal nootropic-ozone therapy. In the control group from 5 (35.7\%) patients after repeated carrying out endolumbal nootropicozone therapy in postoperative period there was not much positive neurological symptomatology and $9(64.3 \%)$ even patients in postoperative period it remained the same. Received in the dynamics of the lumbar puncture data sanation and liquor dynamic tests have shown that improving the patency of liquor content spaces of spinal cord after 2-3 times a endolumbal nootropic-ozone therapy in $70.0 \%$ of cases, and data liqoulogical studies have shown that when endolumbal nootropic-ozone therapy the sanation of liquor was already in the first 3-4 days after injury, while patients control group has the full purifying liquor 8-10 per day. Professionally conducted decompression-stabilizing operations on the spine with the use of endolumbal nootropic-ozone therapy, patients with complicated spinal injury in acute period of injury significantly improves both the results of the treatment and nevrologic symptoms that reduces disability, and this positively affects the quality of life of these traumas.
\end{abstract}

Keywords: Endolumbal Nootropic-Ozone Therapy, Acute Period Spinal Injury, Complex Treatment

\section{Introduction}

Abnormal spinal trauma is the most severe damage to the human musculoskeletal system and is found in the $2.3-4.7 \%$ of cases[1-3]. The lethality of these injuries is up to 25$30 \%[4]$ and disability when it reaches up to $86-94 \%[5-7]$.

In much of the results of treatment depend on the quality of medical aid in acute period of complicated spinal injuries in such cases, the purpose of early and effective treatment of these patients is not only the restoration of the basic function of the spine, but also the development of mechanisms of compensation broken spinal cord functions. Therefore, timely and efficiently provided medical care in specialized institutions significantly improves outcomes of injuries and enhances the quality of life of the victims.

In the last few years to treat complicated injuries of the spine, along with surgical method uses different medications that can help improve metabolism and blood circulation, reduce edema and medications for the injured area of the spinal cord.

So far, have been widely used corticosteroid medications (dexamethasone and methylprednisolone) in acute period of injury. Use of steroid hormones improves metabolism in acute period of spinal cord injury. Corticosteroids also provide stabilizing membranes, reduces edema, "clean" acid radicals, reduce metabolism products contribute to cerebrospinal fluid diffusion of oxygen into the fabric. But 
the drugs have significant side effects, as well as a number of reasons.

Now continue to search for drugs and methods aimed at correlation metabolic violations in the spinal cord, resulting in hypoxia and to improve treatment outcomes.

New approaches to improve the "survival" of nerve structures under hypoxia is the use of catalytic and regulating brain metabolism and thus enhance the ability of nerve cells and its various structures to reparations and resistance to hypoxia. From this point of view the most promising is the use of nootropic drugs.

In the experiment found that nootropic drugs have fairly high activity in different types of tissue hypoxia after injury[7].

Introduction to drugs in cerebrospinal fluid using lumbar puncture has a long history of use in the treatment of certain diseases in recent years, widespread use of been endolumbal introduction of antibacterial means for treating meningitis[8]. E.I. Babichenko (1984), N.M. Majidov (1982) in the agreement means accelerating resorption effusive in subarachnoid cavity blood and warning formation commissure, offered and used the endolumbal introduction lydase (hyaluronidase).

For braking the excess production of kinin in the affected area of the brain in the acute period of craniocerebral injury. E.G. Valeev applied endolumbal introduction contrykal (aprotinin).

Endolumbal introduction of drugs improves the efficiency of treatment that is targeted by medicinal products to the stricken areas of the brain and spinal cord.

In the complex treatment of certain diseases in recent times a significant place on the endolumbal introduction of ozone-oxygen mixture, which increases the redox processes in the brain and spinal cord and brain's commissures drop out in brain tunic.

A.B. Bolgaev (1989) for the prevention and treatment of cerebral cicatrical adhesions processes in the spinal cord in 36 patients with complication of injury lower thoracic and lumbar vertebrae applied endolumbal insufflations of ozone-oxygen mixture, thereby improving the results of the treatment on $22 \%[9,10]$

Investigations and analysis of literature data indicate that the search for new treatments for spinal injury in the acute period to improve its outcomes and prevention cicatrical adhesions of trauma is forward-looking and processes before the end of the solved problem. On the accessible literature we have not met us publications on the use and effectiveness of endolumbal applications, including nootropic and ozone complications of spinal injuries.

The purpose of the study: Is to enhance the effectiveness of the treatment of patients with spinal injury complicated by endolumbal introduction nootropic substances and ozone.

Material and methods: Under our supervision were 25 patients with spinal injury complicated by different localization. Distribution of patients by age and gender is shown in Table 1.
Table 1

\begin{tabular}{lccccccc}
\hline \multirow{2}{*}{ Sex } & \multicolumn{7}{c}{ Age, years } \\
& Up to 14 & $15-20$ & $21-30$ & $31-40$ & $41-50$ & Total \\
\hline Women's & - & 1 & 2 & 3 & 3 & 9 \\
Male & - & 4 & 2 & 4 & 6 & 16 \\
Total & - & 5 & 4 & 7 & 9 & 25 \\
\hline
\end{tabular}

Table 2

\begin{tabular}{cccccccc}
\hline \multirow{2}{*}{ Sex } & \multicolumn{7}{c}{ Age, years } \\
& Up to 14 & $15-20$ & $21-30$ & $31-40$ & $41-50$ & Total \\
\hline Women's & - & - & 1 & 2 & - & 3 \\
Male & - & - & 2 & 3 & 6 & 11 \\
Subtotal & - & - & 3 & 5 & 6 & 14 \\
\hline
\end{tabular}

Of them $19(76.0 \%)$ patients are injured at home, 6 $(24.0 \%)$ in road accident. From 25 patients 16 (64.0\%) admission to hospital in the first 6-12 hours after the injury. All patients with complicated spinal performed spinal cord decompression-stabilizing operations on spine and endolumbal nootropic-ozone therapy. Time of operation from injuries ranged from 8 to 24 hours.

Of all the patients were male $(64.0 \%) 16$, all of them were of working age. Damage the cervical spine was observed in $5(20.0 \%)$ patients, $14(56.0 \%)$ thoracic and lumbar $6(24.0 \%)$ vertebrae. The vast majority of patients observed partial syndrome of spinal cord conduction disorders. Partial violation of the functions of the pelvic organs when observed in all patients. Three patients, who noted compression fracture of body CV, CVI and CVII vertebrae with partial compression of spinal cord anterior decompression operation has occurred-spinal and spondylosyndesis with autotransplant. Two patients, who have had a fracture of the body and posterior structures of $\mathrm{CV}$ and CVI vertebrae carried out anterior decompression and spondylosyndesis with autotransplant, and also decompression laminectomy with posterior spondylosyndesis. All patinets after operation carried out external fixation with Schantz's splint.

All 14 patients with complicated trauma of the thoracic spine performed transthoracic approach anterior decompression of spinal and interbody vertebral autogenous bone graft spondylosyndesis operations. Five of 6 patients with fractures of the body LI and LIII with partial posterior compression of spinal cord performed decompression laminectomy, and one patient anterior decompression of the spinal cord carried out transperitoneal approach and spondylosyndesis with autotransplant, which given from iliac crest.

As the control group were 14 patients with complicated trauma of the spine, which carried out similar operations in the acute period, but without the use of endolumbal nootropic-ozone therapy. It was only a diagnostic and sanation lumbar puncture.

Distribution of patients control group by age and gender is shown in Table 2 .

In this group of patients is also dominated by male 11 $(78.6 \%)$ patients. Damage the cervical spine and spinal 
cord was observed in $3(21.4 \%)$ patients with thoracic 8 $(57.2 \%)$ and from the lumbar from $3(21.4 \%)$ patients. Partial syndrome of spinal cord conduction disturbance was observed in almost all patients. Trophic disturbances as pressure ulcer diagnosed by $6.8 \%$ of patients.

Methods of research. To evaluate the results of treatment we have used traditional clinic neurologic, x-ray and laboratory investigations. From laboratory analysis have been common blood and urine tests, biochemical blood analysis, as well as liquor. For assessing the severity of spinal cord injury classification system used by us on this classification of Frankel. All patients were in the Group C and D.

Indication to the appointment of endolumbal nootropicozone therapy in complicated trauma of the spine are: prevention or reduction of spinal cord edema in traumatic in the first 24 hours after injury or operation improve the metabolism of the spinal cord, improving patency subarachnoid space, dissociation and delamination early arachnoidal commissures, improving blood and luqior circulation in the area of the damaged portion of the spinal cord, improvement of neurological symptoms and prevention of trophic disorders.

Endolumbal nootropic-ozone therapy method in complicated trauma of the spine is deduced the following: on the lateral patient in aseptic conditions under local anaesthesia is lumbar puncture at the level of L III- IV vertebrae special needle, with the assistance of the water pressure is measured by the original liquor pressure and liquor dynamic tests in sterile tube is taken to the common $5 \mathrm{ml}$ liquor then rises head end beds in $300-35$ and puncture needle with syringe endolumbal introduction solution of pyracetam in a dose of 500-1000 mg drug cultivation in saline 1:4 next using another syringe portions 20-40 cm3 ozone. Ozone produced by ozonator. Next endolumbal introduction through 24 hours. The number of introductions to one patient in the course of treatment is based on general and neurological condition of the patient and ranges from 1 to 6 times.

\section{Results and Discussion}

The main criterion for evaluating the effectiveness of this method is clinic neurologic, liquor dynamic and liquorlogical: the emergence of positive neurological symptoms within two weeks after endolumbal nootropicozone therapy. 16 patients $(64.0 \%)$ have after $2-3$ times endolumbal nootropic-ozone therapy has appeared positive neurological symptoms as return of sensation the below level of damage and slight movement in the limbs. In 6 $(24.0 \%)$ patients improved neurological symptoms occurred after 5-6 times the endolumbal nootropic-ozone therapy. The remaining $3(12.0 \%)$ patients even after repeated nootropic-ozone therapy in neurological symptomatology have not improved.

In the control group from $5(35.7 \%)$ patients after repeated carrying out endolumbal nootropic-ozone therapy in postoperative period there was not much positive neurological symptomatology and $9(64.3 \%)$ even patients in postoperative period it remained the same. In addition, these patients were observed violation of the functions of the pelvic organs. All operated patients with wound healing were the primary tension. The endolumbal nootropic-ozone therapy any serious complications in patients were not observed.

Received in the dynamics of the lumbar puncture data sanation and liquor dynamic tests have shown that improving the patency of liquor content spaces of spinal cord after 2-3 times a endolumbal nootropic-ozone therapy in $70.0 \%$ of cases, and data liqoulogical studies have shown that when endolumbal nootropic-ozone therapy the sanation of liquor was already in the first 3-4 days after injury, while patients control group has the full purifying liquor 8-10 per day.

\section{Conclusions}

1. At the same time, on time and professionally conducted decompression-stabilizing operations on the spine with the use of endolumbal nootropic-ozone therapy, patients with complicated spinal injury in acute period of injury significantly improves both the results of the treatment and nevrologic symptoms that reduces disability, and this positively affects the quality of life of these traumas.

2. The liquor dynamic tests have shown that when using the endolumbal nootropic-ozone therapy in postoperative period in patients with complicated spinal injury noticeably increase liquor circulation in traumatized site of spinal cord, confirmed that warns formation of arachnoidal cysts and meningocerebral cicatrix in the field of trauma and trophic disorders.

3. That liqoulogical studies have shown that when the application of endolumbal nootropic-ozone therapy in postoperative period in patients with complicated spinal injury sanation of liquor is already in the first 3-4 days after injury, while patients control group complete purification of liquor was celebrated on 8-10 per day.

\section{References}

[1] Biryuchkov Yu.V. About differential approach and technology operations with compression syndromes./Neurosurgery: scientific-practical magazine.2006. N1. - p. 25 - 29.

[2] Romashov P.P. Clinical assessment outcomes, uncomplicated compression fractures of thoracic and lumbar vertebrae: Dissertation. Candidate of Medical Science: Spb., 1999.-20 page.

[3] Ramih E.A. Evolution spine injury surgery in complex restorative treatment/Surgery of the spine. - No. 1, 2004. 85-92.

[4] Agzamov M.K. "Using ozone therapy in brain injury. 
//Problems of morphology and Parasitology ". /Scientific work. Moscow honey. Academy. them. I.M. Sechenov. Moscow 1991. P. 114-115.

[5] Madyarov S.D. Subarachnoid introduction of ozone in the complex treatment of cerebral leptomeningitis. Materials of the plenum of the society of neurologists and psychiatrists. Tashkent. 1990. 189-191.

[6] Briem D Lehmann W Ruecker AH, Windolf J, Rueger JM, Linhart w. Factors influencing the quality of life after burst fractures of the thoracolumbar transition//Arch Orthop Trauma Surg-Jul 9, 2004. - P. 234-6.

[7] Kaya r.a., Aydin y. Modified transpedicular approach for the surgical treatment of severe thoracolumbar or lumbar burst fractures//Spine. -No. 4 (2), 2004. - P. 208-217.

[8] BolgaevA.B. "Complex treatment of uncomplicated and complicated fractures lowers thoracic and lumbar vertebrae." Dissertation. Doctor of Medical Science. Tashkent. 1989

[9] Valeev E.K. Clinic and treatment heavy brain injury in acute period. "Dissertation. Doctor of Medical Science. Kazan. 1987.

[10] Lebedova N.V. "Nootropil in neurology". "Pharmacology nootropic agents." "Experimental and clinical study". Moscow. 1986. 125-128. 